\title{
A parametric study of 3D printed polymer gears
}

\author{
Ye Zhang ${ }^{1} \cdot$ Ken Mao $^{1} \cdot$ Simon Leigh ${ }^{1} \cdot$ Akeel Shah $^{1} \cdot$ Zhiming Chao $^{1} \cdot$ Guotao Ma $^{1}$
}

Received: 12 September 2019 / Accepted: 30 March 2020 / Published online: 28 April 2020

(C) The Author(s) 2020

\begin{abstract}
The selection of printing parameters for 3D printing can dramatically affect the dynamic performance of components such as polymer spur gears. In this paper, the performance of 3D printed gears has been optimised using a machine learning process. A genetic algorithm (GA)-based artificial neural network (ANN) multi-parameter regression model was created. There were four print parameters considered in 3D printing process, i.e. printing temperature, printing speed, printing bed temperature and infill percentage. The parameter setting was generated by the Sobol sequence. Moreover, sensitivity analysis was carried out in this paper, and leave-one cross validation was applied to the genetic algorithm-based ANN which showed a relatively accurate performance in predictions and performance optimisation of 3D printed gears. Wear performance of 3D printed gears increased by 3 times after optimised parameter setting was applied during their manufacture.
\end{abstract}

Keywords 3D printing $\cdot$ Nylon $\cdot$ Gears $\cdot$ Wear $\cdot$ Polymer gears $\cdot$ Machine learning

\section{Introduction}

For applications such as automotive and aerospace engineering, polymer gears have unique advantages over metal gears: low cost and weight, high efficiency, quietness of operation, functioning without external lubrication, etc. The performance of $3 \mathrm{D}$ printed gear has been investigated previously. According to Ye et al. [1], 5 different 3D printing nylon materials have been compared; result shows Nylon 618 has outstanding performance compared with other nylon materials, including $23 \%$ carbon fibre reinforced nylon filament. There

\section{Ye Zhang}

ye.zhang@warwick.ac.uk

Ken Mao

k.mao@warwick.ac.uk

Simon Leigh

s.j.leigh@warwick.ac.uk

Akeel Shah

akeel.shah@warwick.ac.uk

Zhiming Chao

z.chao@warwick.ac.uk

Guotao Ma

g.ma.1@warwick.ac.uk

1 School of Engineering, The University of Warwick, Coventry CV4 7AL, UK are many investigations into the characteristics of wear and thermal behaviour of injection-moulded gears. Mao et al. [2] carried out an analysis of the friction and wear behaviour of acetal and nylon gears including characterising the failure mechanism and thermal analysis. The results showed the operational time of polymer spur gears under different circumstances. Hu and Mao [3] investigated the effects of different misalignments on the fatigue of polymer gears during use. Hooke et al. [4] proved that increases in the surface temperature can dramatically increase the wear rate of the gear tooth. Moreover, Gauvin et al. [5] carried out an investigation into the maximum surface temperature experienced by polymer gears without lubrication. Mao et al. [6] introduced a new method to predict the surface temperature of acetal gears and found the correlation between fatigue life and tooth size. Additive manufacturing (AM) and $3 \mathrm{D}$ printing processes have become increasingly popular; the applications of 3D printing are usually suitable for relatively low production volumes, small size parts and complex designs. It is generally understood that $3 \mathrm{D}$ printing is cost effective if production volumes are below 1000 units in comparison with plastic injection moulding [7]. The technology has been applied in wide range of industries, including the automotive industry, aerospace, medical and architectural [8]. There was limited research on dynamic performance of 3D printed polymer parts; however, there are several investigations regarding the parameters which affect the mechanical and thermal properties. Chacon et al. [9] has investigated the effect of process parameters on 
mechanical performance of PLA in terms of on-edge orientation, layer thickness and feed rate. It has been shown that higher printing speeds can increase the mechanical performance of printed parts. Giovanni [10] carried out Taguchi's experimental design for fatigue analysis of PLA and claimed that infill percentage had the most influence on fatigue life. Kuznetsov et al. [11] claimed that printing temperature and printing speed could dramatically dominate the mechanical properties of the 3D printed part. Moreover, the thermal conductivity of 3D printing filaments can also affect the properties of the object [12], increasing or decreasing the bonding quality between each layer during fused deposition modelling $[13,14]$. In order to understand the complicated interplay between these different process parameters and to select the most appropriate parameter set for the production of $3 \mathrm{D}$ printed gears, a multiple regression process is required.

There was very limited research regarding of machine learning associated with predicting performance of gears and only some on its application to $3 \mathrm{D}$ printing processes. Fracture behaviour of 3D printed material has shown dramatically different compared with other materials [15]. Deng et al. [16] introduced optimisation methods to the multi-factor printing of a ceramic slurry by using artificial neural networks. Koeppe et al. [17] used neural networks to analyse load distribution in 3D printed lattice cell structures. Delli and Chang [18] used supervised machine learning to do real time monitoring of 3D printing to eliminate printing time and waste. Those research reports have provided valuable results in terms of static force analysis and monitoring of the $3 \mathrm{D}$ printing; however, dynamic analysis of 3D printed parts require further investigations. $\mathrm{Li}$ et al. [19] has introduced a method using support vector machine to predict dynamic contact characteristics for helical gears. Sun et al. [20] used neural networks to optimise and predict a gear hobbing process to improve the efficiency and reduce the cost. Sun et al. [21] used artificial neural networks and support vector machines with genetic algorithms to monitor the faults in gears. To find the correlation of $3 \mathrm{D}$ printing process parameters and dynamic performance of polymer gears would be a significant benefit to researchers both in the fields of 3D printing and gear manufacture to increase the efficiency of the 3D printing process and quality of the resultant $3 \mathrm{D}$ printed spur gears.

Performing multi-parameter regression has many challenges, for example missing data and data noise, as well as high dimensionality which impacts the ability to identify the relations between parameters [22]. Through ordinary mathematical solutions, it is computationally complex to solve multi-target modelling, and targets often may not correlate. However, by using some baseline methods, such as Gaussian processes, neural networks or support vector machines, the complexity of the problem can be much reduced [23].

\section{Methods and experiments}

All 3D printing parameters were set as default and printed with manufacturer-recommended printing temperature $\left(250^{\circ} \mathrm{C}\right)$, bed temperature $\left(30^{\circ} \mathrm{C}\right)$ and speed $(45 \mathrm{~mm} / \mathrm{s})$ apart from infill percentage, which was set to $60 \%$ for both printer systems. Under initial setting which printing temperature was $245^{\circ} \mathrm{C}$, printing speed was $45^{\circ} \mathrm{C}$, infill percentage was $60 \%$ and bed temperature was $45^{\circ} \mathrm{C}$ gear running under $10 \mathrm{Nm}$ torque and last around $16 \mathrm{~h}$. There are several stages required in order to complete dynamic performance optimisation of 3D printed gears. First of all, the use of a Sobol sequence was employed to generate Sobol random parameters with multiple data points per parameter. The Sobol sequence is a low discrepancy quasi-random sequence. Using this method, a set of test data comprising of 50 points was generated, including a printing temperature range $\left(230-275^{\circ} \mathrm{C}\right)$, printing speed range $(20-75 \mathrm{~mm} / \mathrm{s})$, infill percentage (20-80\%) and bed temperature range $\left(30-70^{\circ} \mathrm{C}\right)$. Bed temperature refers to the temperature of printing surface which will affect the first few layers during printing. Infill percentage represents how 'hollow' the gear is, with the aim of reducing the infill percentage and hence reducing the weight and inertia during operation. Each parameter was increased by factor of one, for example printing temperature was increased from 230 to $275^{\circ} \mathrm{C}$ and the Sobol sequence covers the entire range, hence there was a total of 45 data points for printing temperature. Figure 1 shows the specification of gears.

Furthermore, by applying a similar range for each parameter, a small number of experiments could potentially provide insights into the complex combination of each test data category, roughly reducing a total of $45 \times 45 \times 40 \times 60=5,940,000$ possible combinations of 3D printing settings to a sample set of 50 chosen by the Sobol sequence. Gears were produced using an Ultimaker 3 extended fused deposition modelling (FDM) system. Gears were printed on a tufnol print surface due to superior adhesion between the nylon and tufnol, eliminating the peel off during the 3D printing process. A total of 100 gears were printed (50 matched pairs) with an average printing time of around $6 \mathrm{~h}$ per gear (depending on the setting of the parameters). After printing, gears were mounted on a gear wear test rig subject to the gear performance life cycle with $10 \mathrm{Nm}$ torque. The tests included the recording of the wear occurring at the gear tooth and showed the different stages of gear operation until gear failure. The time from commencing the test run on the test rig until the gear failure was considered as the fatigue time. After acquiring the test data for the printed gears, the $3 \mathrm{D}$ printing parameters were used as input, and the corresponding gear life cycle data from test rig was used as an output to create a neural network model of correlations between input and output. The Gaussian process was also employed to perform multi-parameter regression to find out the approximate likelihood of output accuracy. By using the model generated by the artificial neural network (ANN) and GP, a subsequent sensitivity analysis was carried out to investigate the 
Fig. 1 a 3D printed nylon gear cross section in simplified 3D. $\mathbf{b}$ Specifications of gears

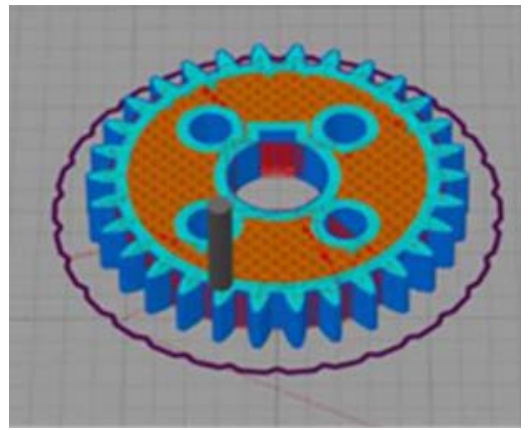

\begin{tabular}{ll}
\hline Module & $2 \mathrm{~mm}$ \\
\hline Tooth number & 30 \\
Pressure angle & $20^{\circ}$ \\
Face width & $15 \mathrm{~mm}$ \\
Nominal backlash & $0.18 \mathrm{~mm}$ \\
Tooth thickness & $3.14 \mathrm{~mm}$ \\
Contact ratio & 1.65 \\
\hline
\end{tabular}

(a)

(b) relations of each multi-parameter. The process was shown in Fig. 1. Table 1 shows the result of the tests via the different Sobol sequence settings.

\subsection{Sobol sequence}

The Sobol sequence is a method to sample data in a quasirandom sequence, in which data was selected in a uniformly random form. The Sobol sequence was first introduced by Russian mathematician I.M Sobol [24]. According to Savine, Sobol's sequence could provide better evenness and higher speed to fill the space within a hyper cube [25]. Sobol's sequence had over past 20 years of improvement of the algorithm to capable applied to high dimension. Hence, Sobol's sequence became a best practice in different applications. Sobol's sequence was generated with Sobol's generator fitted in MATLAB; experiment data of each parameter was generated based on the algorithm of Sobol's sequence. This code below creates 50 vectors ( 4 components in each vector), according to a 4-variate uniform distribution implemented (approximately) using the Sobol sequence. Each component in each vector is a number between 0 and 1 . The command above produces a matrix ' $\mathrm{X}$ ' that lines up each of the vectors as a column in the matrix $X$. There are therefore 50 columns and 4 rows.

Temperature $=225+X(1,:) * 50$

This takes the first component of each of the 50 vectors and rescales it to get a temperature input value (between 225 and $275 \mathrm{~K})$. Basically, use the first row $X(1,:)$ of $X$.

Speed $=20+X(2,:) * 50$

Same as above but use the 2 nd row $X(2,:)$ of $X$ to get the printing speed values (between 20 and $70 \mathrm{rpm}$ ).

BedT $=10+X(3,:) * 50$

3rd row $X(3,:)$ of $X$ to get the bed temperature values

Infill $=20+X(4,:) * 50$ 4th row $X(4,:)$ of $X$ to get the infill values

Input(:,1) $=$ Temperature'

Create a matrix called 'Input' and make the first column as the 50 temperature values by typing the above (you need to transpose the vector of temperature values by using a prime, i.e. ').

Input $(:, 2)=$ Speed $^{\prime}$

Second column of input is the speed value.

$\operatorname{Input}(:, 3)=\operatorname{BedT}^{\prime}$

Third column of input is the bed temperature values.

$\operatorname{Input}(:, 4)=\operatorname{sInfill}^{\prime}$

Fourth column of input is the infill values.

\subsection{Test rig}

The test rig was designed to test the gear wear whilst the gears were meshed and running. Details of the test rig employed have been discussed in previous paper [1,2]. 3D printed gears can be tested in much the same way as metal gears, using a back to back test configuration where the gears are loaded by winding in the torque to a prescribed level [2]. A torque of $10 \mathrm{Nm}$ was added to the gears, with each test gear operated until failure. A motor was used to drive gears with an externally applied torque, with the reaction force between gear teeth were equivalent to the bearing block and loading arm (Fig. 2). This loading method permits large amounts of wear without significantly affecting the applied torque (Fig. 3).

\subsection{Artificial neural networks}

Artificial neural networks simulate the physiological structure and mechanism of the human brains in order to solve complex problems. It is a machine learning process which is distinctly different from common methods such as signal reasoning and 
Table 1 Input parameters generated by the Sobol sequence and output from test rig

\begin{tabular}{|c|c|c|c|c|c|}
\hline Testing number & Printing temp $\left({ }^{\circ} \mathrm{C}\right)$ & Printing speed $(\mathrm{m} / \mathrm{s})$ & Bed temperature $\left({ }^{\circ} \mathrm{C}\right)$ & Infill percentage $(\%)$ & Test result, gear fatigue time (hours) \\
\hline 1 & 230 & 25 & 30 & 20 & 0.04 \\
\hline 2 & 253 & 50 & 50 & 50 & 20 \\
\hline 3 & 264 & 38 & 60 & 35 & 11.11 \\
\hline 4 & 241 & 63 & 40 & 65 & 30 \\
\hline 5 & 247 & 44 & 55 & 28 & 1.94 \\
\hline 6 & 269 & 69 & 35 & 58 & 24.69 \\
\hline 7 & 258 & 31 & 45 & 43 & 9.32 \\
\hline 8 & 236 & 56 & 65 & 73 & 21.03 \\
\hline 9 & 238 & 41 & 43 & 61 & 15.57 \\
\hline 10 & 261 & 66 & 63 & 31 & 10.1 \\
\hline 11 & 272 & 28 & 53 & 76 & 30.18 \\
\hline 12 & 250 & 53 & 33 & 46 & 20.6 \\
\hline 13 & 244 & 34 & 68 & 54 & 10.12 \\
\hline 14 & 267 & 59 & 48 & 24 & 6.66 \\
\hline 15 & 255 & 47 & 38 & 69 & 12.9 \\
\hline 16 & 233 & 72 & 58 & 39 & 0.36 \\
\hline 17 & 234 & 48 & 64 & 44 & 12.77 \\
\hline 18 & 257 & 73 & 44 & 74 & 36.8 \\
\hline 19 & 268 & 36 & 34 & 29 & 1.65 \\
\hline 20 & 245 & 61 & 54 & 59 & 16.66 \\
\hline 21 & 251 & 30 & 49 & 37 & 2.88 \\
\hline 22 & 274 & 55 & 69 & 67 & 20.16 \\
\hline 23 & 262 & 42 & 59 & 22 & 2.67 \\
\hline 24 & 240 & 67 & 39 & 52 & 10.32 \\
\hline 25 & 237 & 33 & 51 & 71 & 12.24 \\
\hline 26 & 260 & 58 & 31 & 41 & 1.96 \\
\hline 27 & 271 & 45 & 41 & 56 & 7.28 \\
\hline 28 & 248 & 70 & 61 & 26 & 0.06 \\
\hline 29 & 243 & 39 & 36 & 78 & 21.24 \\
\hline 30 & 265 & 64 & 56 & 48 & 27.78 \\
\hline 31 & 254 & 27 & 66 & 63 & 25.71 \\
\hline 32 & 231 & 52 & 46 & 33 & 0.39 \\
\hline 33 & 232 & 38 & 54 & 55 & 25.2 \\
\hline 34 & 255 & 63 & 34 & 25 & 11.38 \\
\hline 35 & 266 & 26 & 44 & 70 & 8.4 \\
\hline 36 & 243 & 51 & 64 & 40 & 1.76 \\
\hline 37 & 249 & 32 & 39 & 62 & 5.16 \\
\hline 38 & 271 & 57 & 59 & 32 & 4.17 \\
\hline 39 & 260 & 45 & 69 & 77 & 34.49 \\
\hline 40 & 238 & 70 & 49 & 47 & 15.67 \\
\hline 41 & 241 & 29 & 62 & 28 & 0.07 \\
\hline 42 & 263 & 54 & 42 & 58 & 14.79 \\
\hline 43 & 274 & 41 & 32 & 43 & 3.06 \\
\hline 44 & 252 & 66 & 52 & 73 & 30.45 \\
\hline 45 & 246 & 48 & 47 & 21 & 0.04 \\
\hline 46 & 269 & 73 & 67 & 51 & 12.77 \\
\hline 47 & 257 & 35 & 57 & 36 & 16.38 \\
\hline 48 & 235 & 60 & 37 & 66 & 32.77 \\
\hline 49 & 234 & 37 & 41 & 79 & 25 \\
\hline
\end{tabular}


Table 1 (continued)

\begin{tabular}{llllll} 
Testing number & Printing temp $\left({ }^{\circ} \mathrm{C}\right)$ & Printing speed $(\mathrm{m} / \mathrm{s})$ & Bed temperature $\left({ }^{\circ} \mathrm{C}\right)$ & Infill percentage $(\%)$ & Test result, gear fatigue time (hours) \\
\hline 50 & 256 & 62 & 61 & 49 & 25.41 \\
\hline
\end{tabular}

logical thinking approaches [26]. ANN is an appropriate method for solving incomplete associative memory, defect characteristic pattern recognition and automatic learning [27]. There are three main reasons that ANN was selected for this research; first of all, the calculation speed of the ANN is significantly computationally cheaper than other methods [28]. Secondly, ANN has strong fault-tolerant ability to minimise the uncertainty during the experiments. Thirdly, ANN is adept in addressing problems with multi-parameter regression, which is hard to solve with purely numerical methods [29]. Back-propagation (BP) training algorithm is the most frequently used ANN training method [30].

\subsubsection{Back-propagation networks}

The principles of the back-propagation networks The detailed stages of BP training method are the following: (1) The sample data for training are input to the network. (2) Data moves forward from input stage to each hidden layer until the output stage, then the output data is generated. (3) The difference between input data and output data is compared, and if the differences are larger than expected, they will be transferred back to the hidden layer. (4) The weight of each neuron is adjusted based on the deviation via the steepest descent method that means calculating the minimum value (maximum value) of the loss function along the gradient descent (ascent) direction and the deviation transited to the input layer. (5) The value proceeds forward again and after repeated iteration; the error constantly diminishes. (6) The training process is over when the gap between the input value and output value is smaller than the expected value.

Figure 4 shows the structure of the ANN model. The ANN model in this paper was carried out based on MATLAB neural network toolbox. Moreover, there is a loop fitted in the model aimed to select optimised hidden number of neural from 1 to 20. Result shows 5 hidden sizes providing less error. The ANN model in this paper is composed of 4 input layer nodes, 5 hidden layer nodes and 1 output layer nodes. The initial parameters of ANN, such as the connection weights between input layer, hidden layer and output layer, and threshold value of hidden layer and output layer have large influence on the predictive performance. Due to the small number of training data, best validation performance could occur at epoch 1 as shown in Fig. 5.

\subsection{Genetic algorithm}

For the traditional ANN predictive models, without combining optimization algorithms, the initial parameters are determined randomly, which is inefficient or prone to converging to local optima, slow convergence speed, overtraining, subjectivity in the determining of model parameters and often pose a convergence problem [31]. The optimised algorithm GA is able to optimise the initial parameters of machine learning models to increase the estimating accuracy and accelerate the convergence speed of the ANN models [32, 33].

Genetic algorithm (GA) is a parallel random search optimisation algorithm to simulate the genetic mechanism of natural GA, and biological evolution GA can conduct efficient heuristic

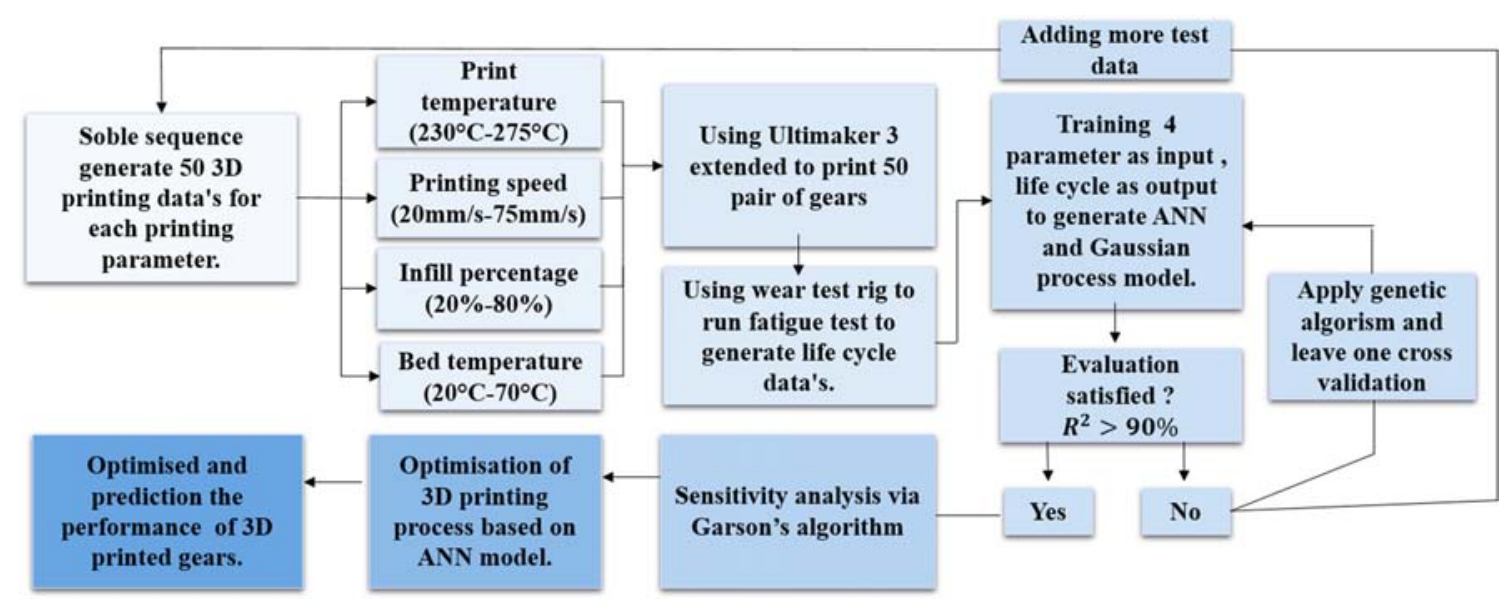

Fig. 2 Schematic of 3D printing optimising process 
Fig. 3 Schematic of wear test rig

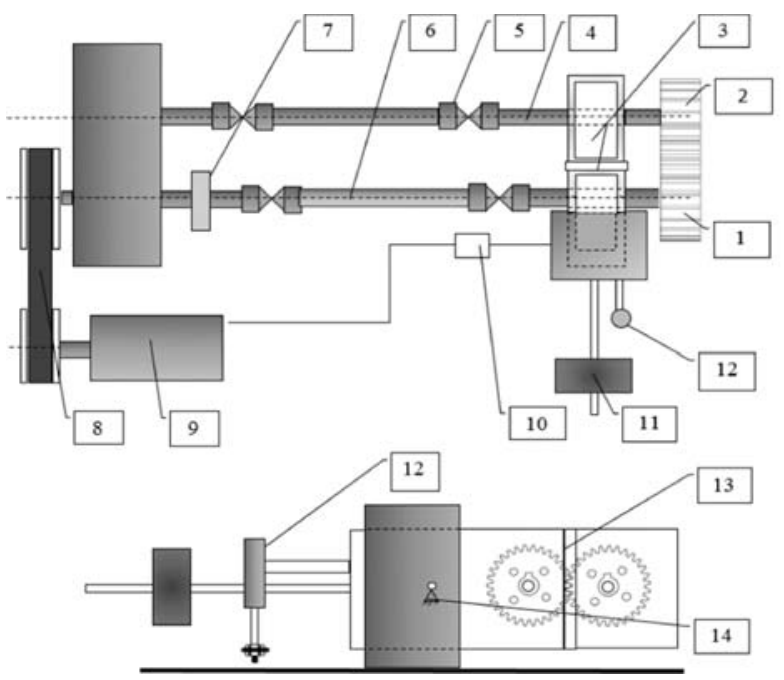

1. Driver gear 8. Pulley

2. Driven gear 9. Motor

3. Pivot block 10. Motor

assembly. controller

4. Driven shaft 11 . Weight

couplings.

\begin{tabular}{ll}
\hline 6. Driving shaft & $\begin{array}{l}\text { 13. Centre } \\
\text { spacer }\end{array}$ \\
\hline 7. Conical clutch & 14. Pivot
\end{tabular}

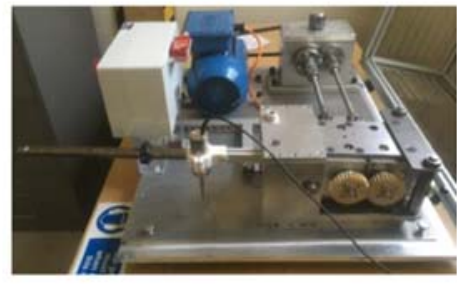

search and parallel computing [34]. It introduces the biological evolutionary principle of 'survival of the fittest' in the coded tandem population formed by optimisation parameters and chooses individuals according to the fitness function of the individuals and the operations of selection, cross and mutation to make the individuals with high fitness value be retained; the individuals with low fitness be eliminated [35]. The new generation would inherit the information of the previous generation and be superior to the previous generation. This iteration is repeated until the predetermined expired criterion is met [36].

The basic operations of the GA are divided into:

\subsubsection{Select operation}

The selection operation refers to the selection of individuals from the old generation to the new generation [37]. The probability that the individual is selected from the old generation to the new generation is related to the fitness value of the individual. The better the individual fitness value, the higher the probability of being selected [38].

\subsubsection{Cross operation}

The cross operation refers to the selection of two individuals from the old generation to produce new individuals by randomly exchanging and combination of the chromosomal locations of the two old individuals [39].

\subsubsection{Mutation operation}

The mutation operation refers to the selection of an individual from the old generation and choosing a point in the chromosome of the individual to mutate to produce a new individual. The basic process of GA is shown in Fig. 6.
Fig. 4 Schematic structure of ANN model

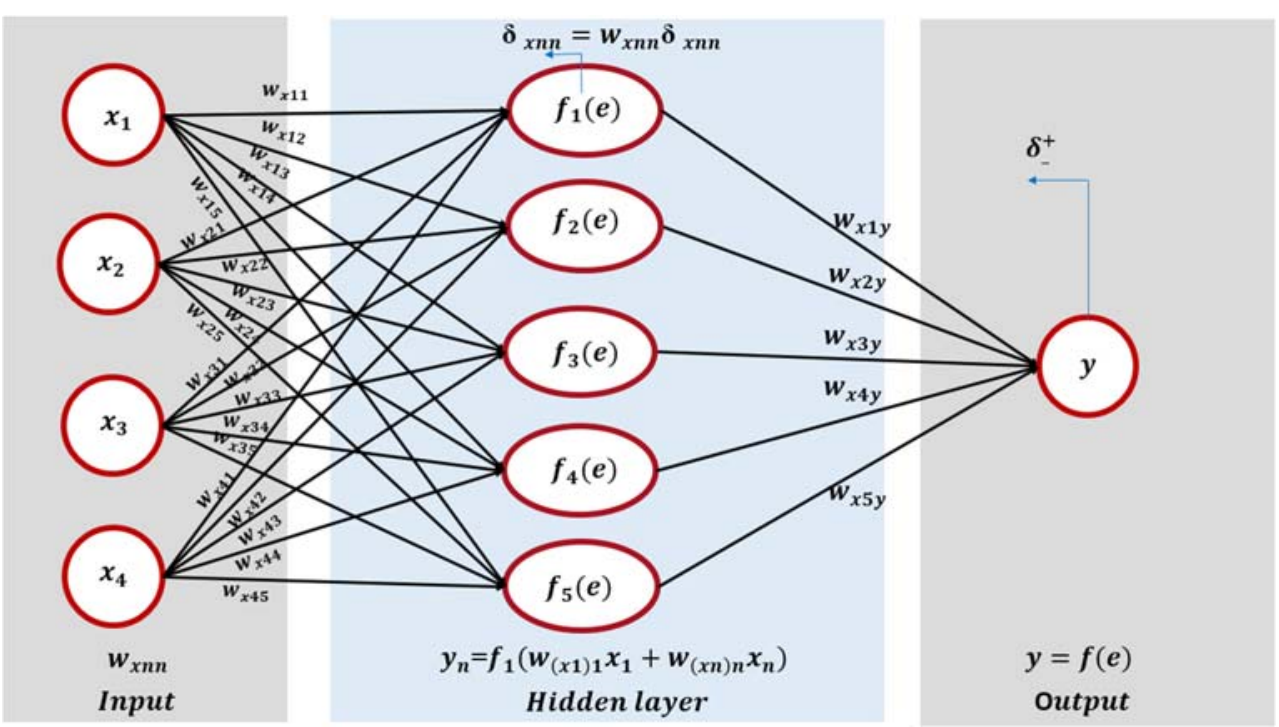




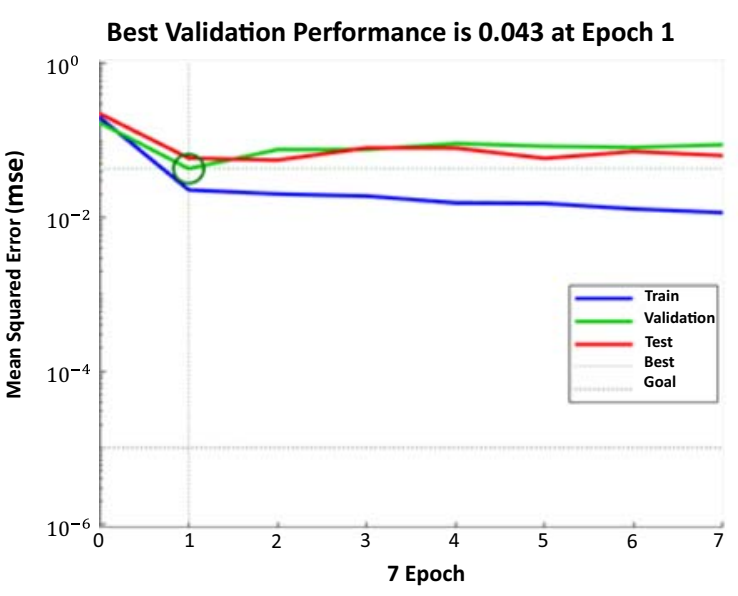

(a)

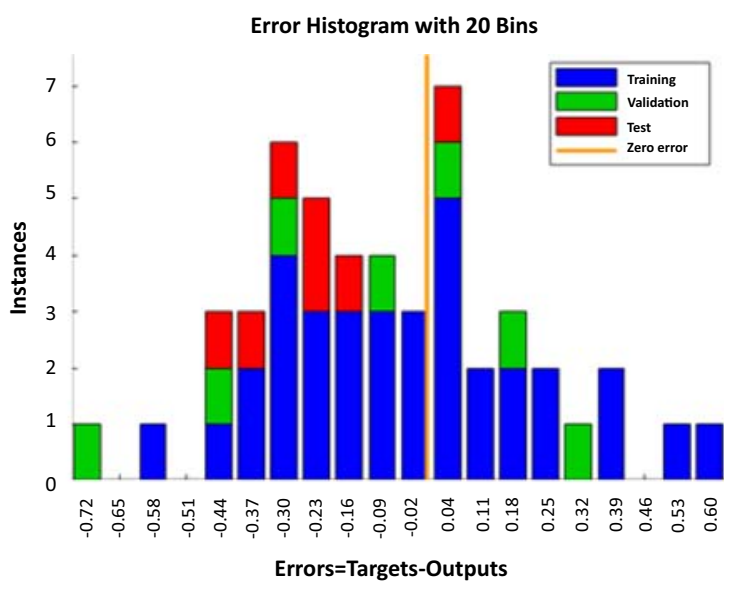

(b)
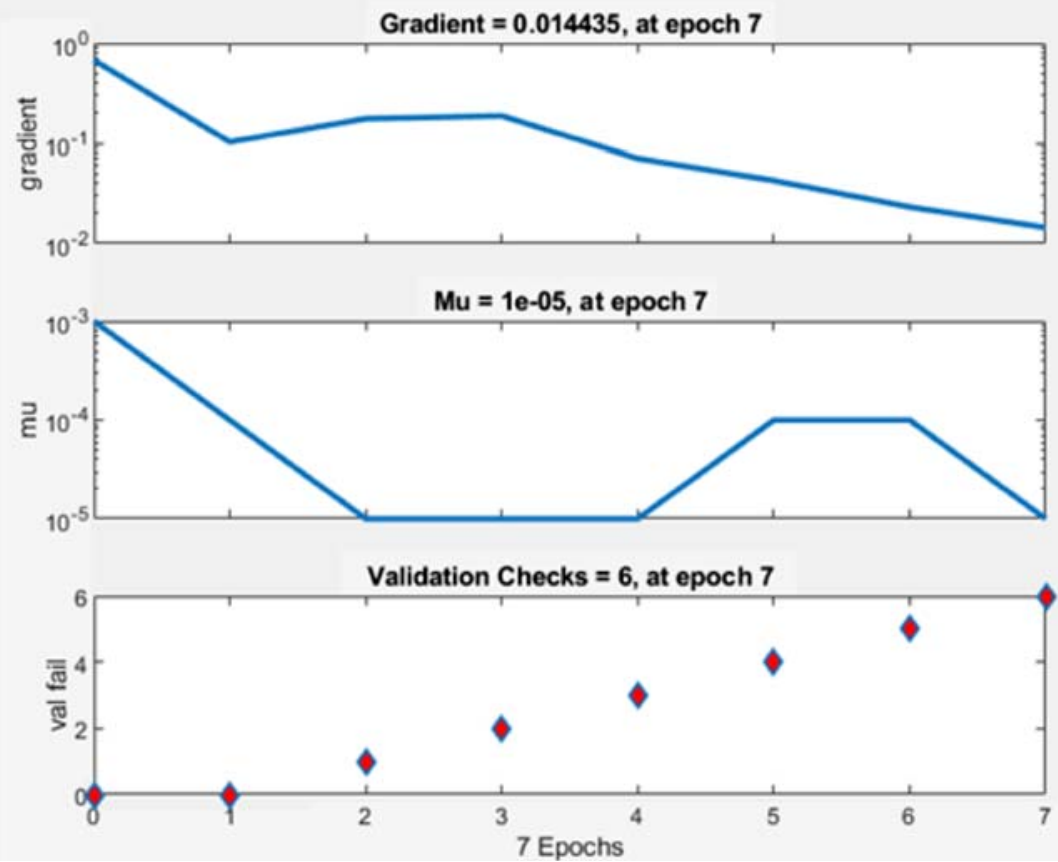

(c)

Fig. 5 Performance validation of ANN

The detailed method of applying GA in improving the performance of ANN is following: the GA is used to optimise the initial parameters of ANN. Each particle in GA contains all information of the initial parameters of the ANN model. According to the fitness function of the individuals and the operations of selection, cross and mutation to make the individuals with high fitness value be retained, the individuals with low fitness are eliminated. This iteration is repeated until the predetermined expired criterion is met. The initial parameters of the particle with the highest fitness are assigned to the ANN model. The objective function (fitness function) is the $R$-squared. The crossover coefficient of the GA algorithm is 0.2 , the mutation coefficient is 0.2 , the size of population is 100 and the maximum iteration number is 100 .

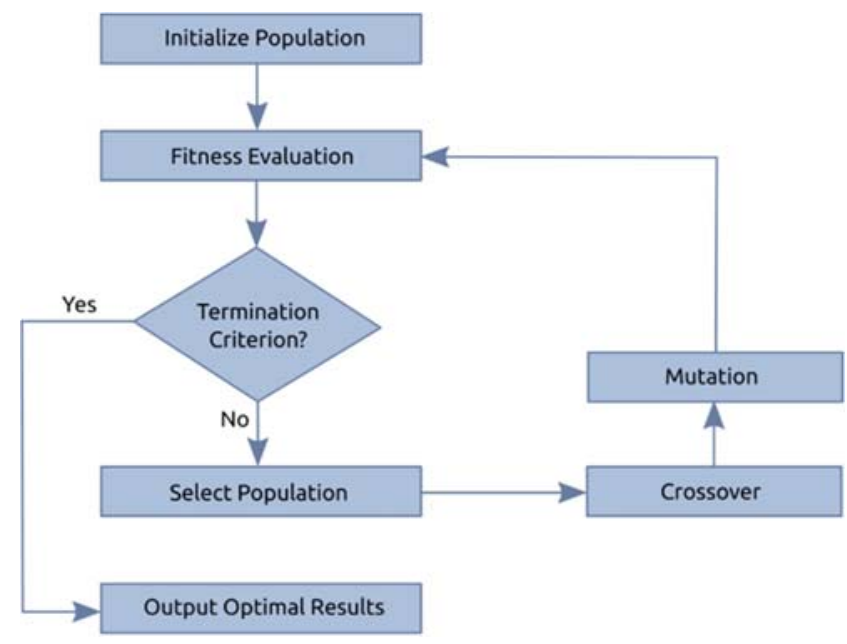

Fig. 6 Schematic of GA process 


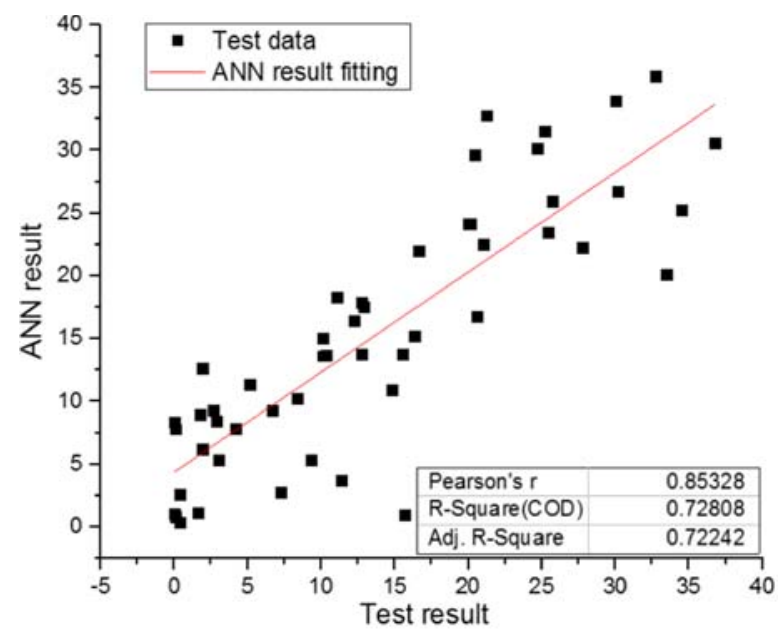

(a)

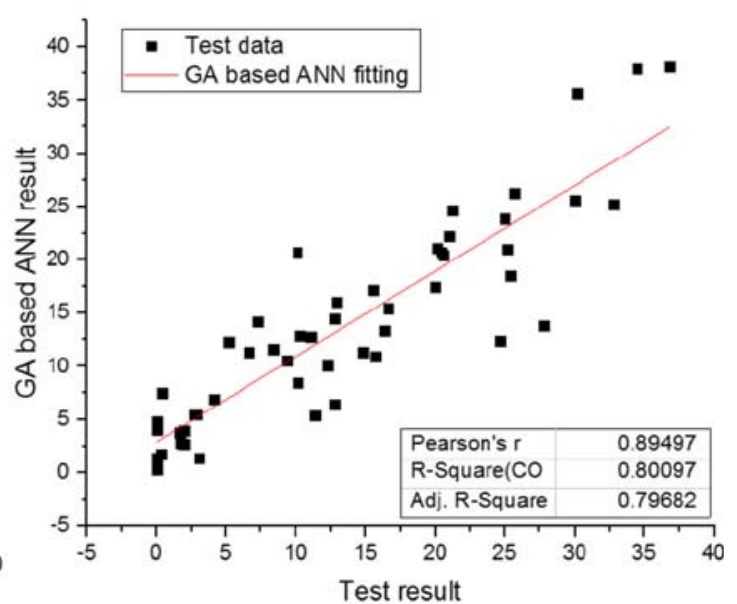

(b)

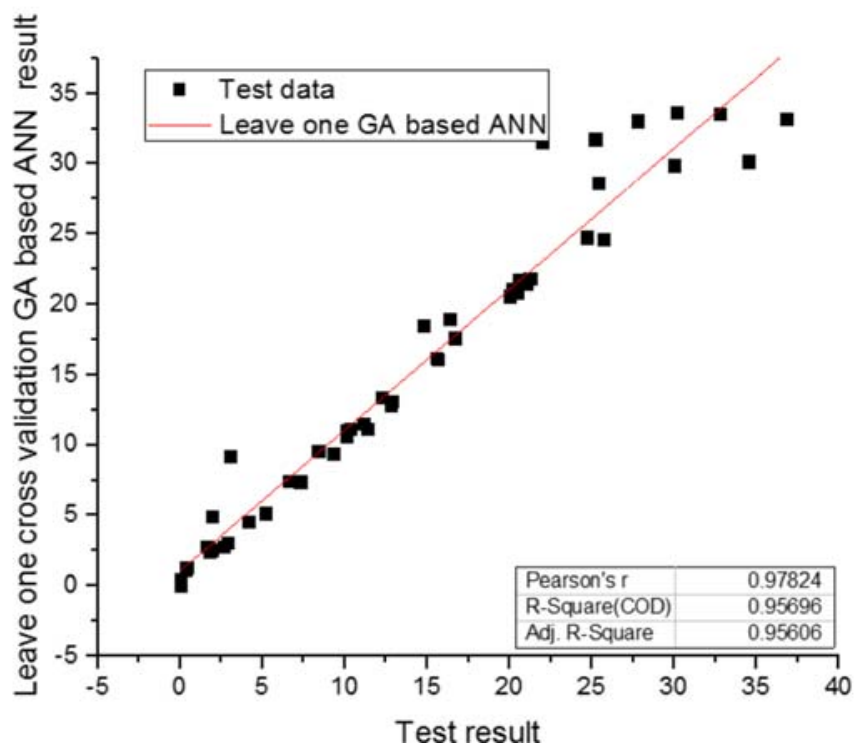

(c)

Fig. 7 Performance result fitted with test data

Fig. 8 Optimisation process of GA on weight ratio of ANN

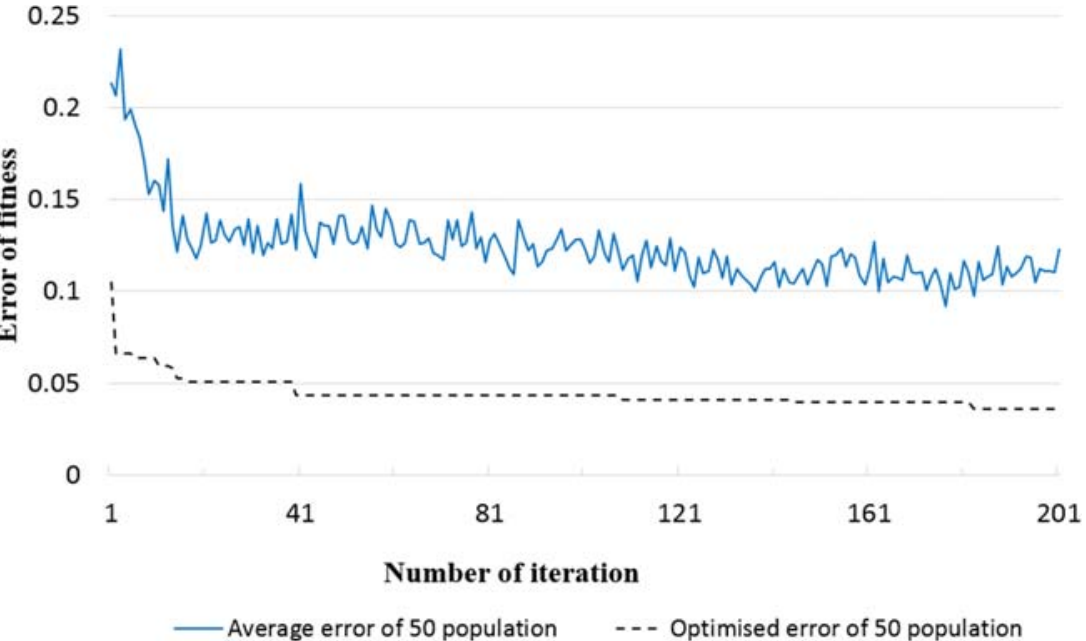


Fig. 9 Performance of different models compared with test result

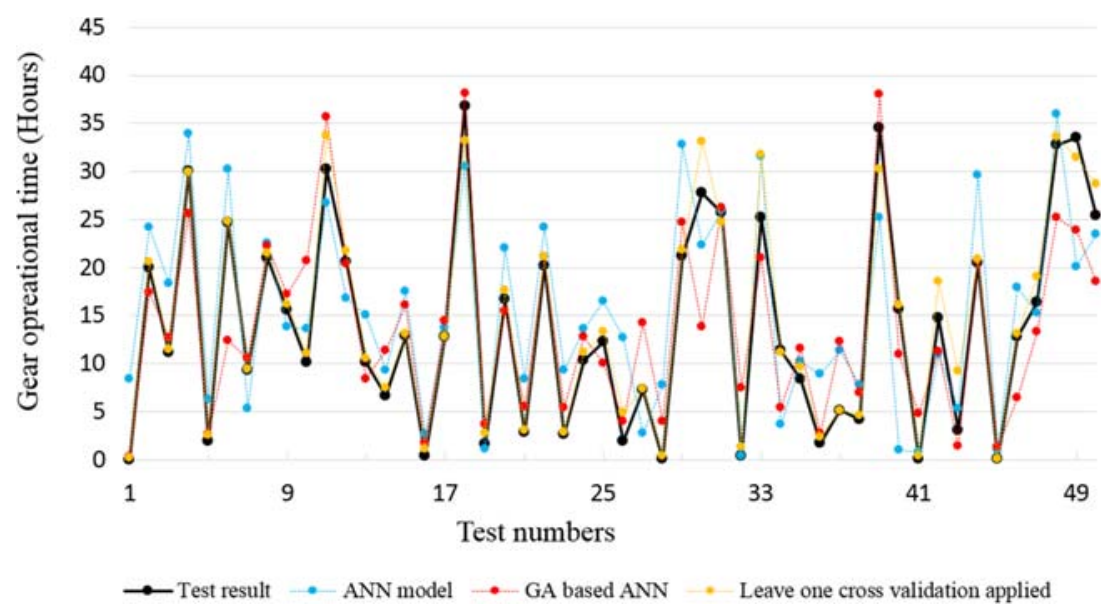

\subsection{Leave-one-out cross validation}

Leave-one-out cross validation is a method which evaluates the performance of a machine learning algorithm, which in this case is an ANN. As a technique, it can increase the prediction accuracy by increasing the training data point to and decrease the test data point to 1 . Hence, leave-one-out cross validation could eliminate the randomness of dividing instances into for training and testing. By changing the ratio of training and testing of AAN, it could maximised the training algorithm to provide a better understanding of model and clearer pattern of the Sobol sequence [40]. Due to the small amount of data, it is workable to maximise the number of the training data.

\subsection{Garson's algorithm}

Based on the established machine learning models, the sensitivity analysis of the input parameters is conducted by adopting Garson's algorithm. In 1991, Garson proposed Garson's algorithm [41, 42], later modified by Goh, for determining the relative importance of the input parameters to the output parameter [39, 41, 43], the equation of Garson's algorithm is shown in Eq. 1; the results of the sensitivity analysis by using Garson's algorithm is shown in Fig. 9.

$R_{\mathrm{ij}}=\frac{\sum_{j=1}^{L}\left(\left|W_{i j} W_{j k}\right| / \sum_{r=1}^{N}\left|W_{r j}\right|\right)}{\sum_{i=1}^{N} \sum_{j=1}^{L}\left(\left|W_{i j} W_{j k}\right| / \sum_{r=1}^{N}\left|W_{r j}\right|\right)}$

where $R_{\mathrm{ij}}$ is the relative importance of input parameters, $W_{i j}, W_{j k}$ are the connection weights of the input layer hidden layer and the hidden-output layer, $i=1,2 \ldots . N, k=1,2 \ldots . M$ ( $\mathrm{N}$ and $M$ are the numbers of the input parameters and output parameters).

\section{Result and discussion}

Figure 7 shows the performance of each model fitting with original test data. Figure $7 \mathrm{a}$ shows the linear fitting between the ANN model and test data given a Pearson productmoment correlation coefficient of 0.85326 and $R$ square of 0.728 . It shows high correlation related to the original test data $[44,45]$. However, to optimise the performance, it is possible
Fig. 10 Sensitivity response contributes to result

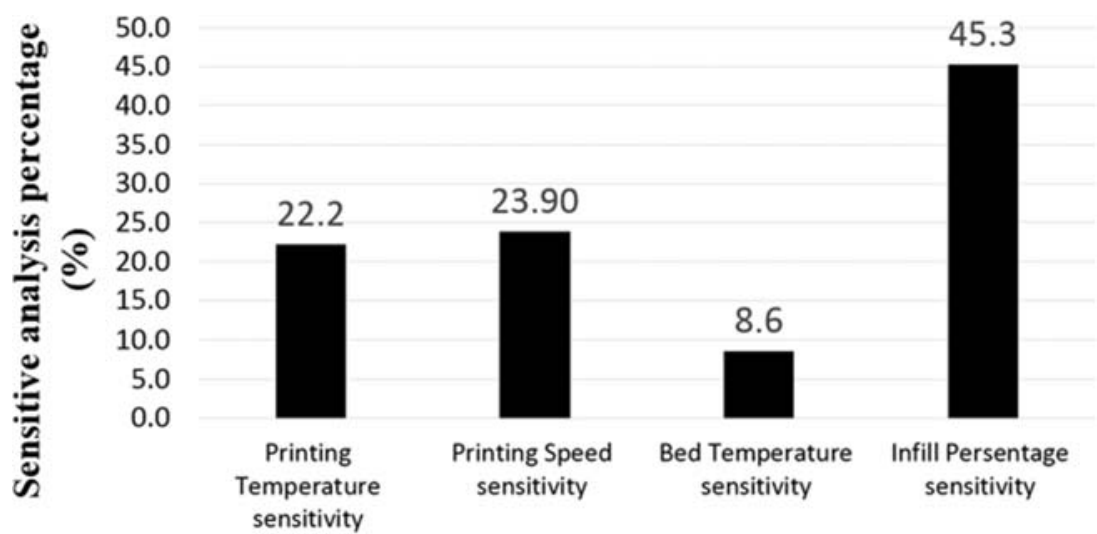

Input Paremeters 
Fig. 11 3D printing process optimisation by ANN simulation

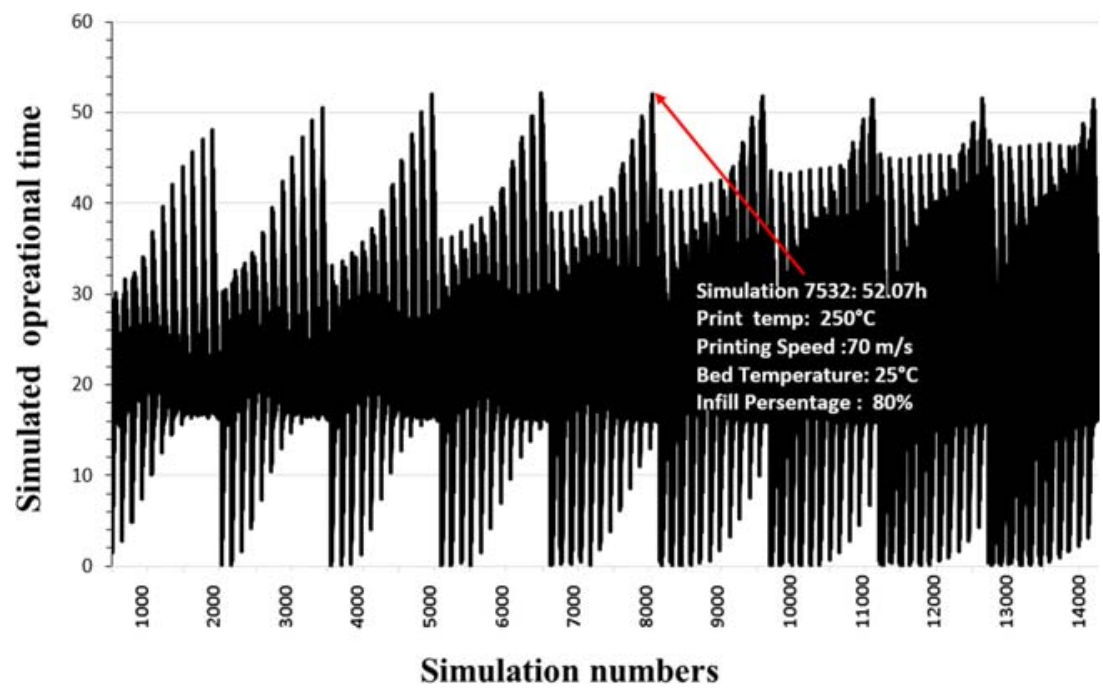

to increase the accuracy of the prediction model. Hence, the GA-based ANN (Fig. 7b) has been applied to the model which yields closer agreements between the measured and predicted values of gear fatigue time. $R^{2}$ increases from 0.728 to 0.8 when the GA is applied; moreover, Pearson's $r$ increased by nearly $5 \%$. This result could be explained by the fact that the proposed ANN-based predictive model accuracy in this case was increased with GA optimisation. Furthermore, the initial target was to achieve an $R$-squared value greater than 0.9 ; hence, the GA-based ANN can provide a relatively satisfactory result. However, optimisation and prediction accuracy can be further increased by applying leave-one cross validation.

Figure 4c shows the model applied with both GA and leave-one cross validation, Pearson's $r$ and $R^{2}$ dramatically increase from 0.83 to 0.97 and 0.728 to 0.956 , respectively. Hence, a model with applied leave-one cross validation was selected as the final model to carry out further analysis.

Figure 5 shows the result of optimisation performed by GA, which is used to optimise the ratio between $\omega$ and $\delta$ in order to improve the accuracy of the ANN. The solid plot represents the average error corresponding to the real test data.

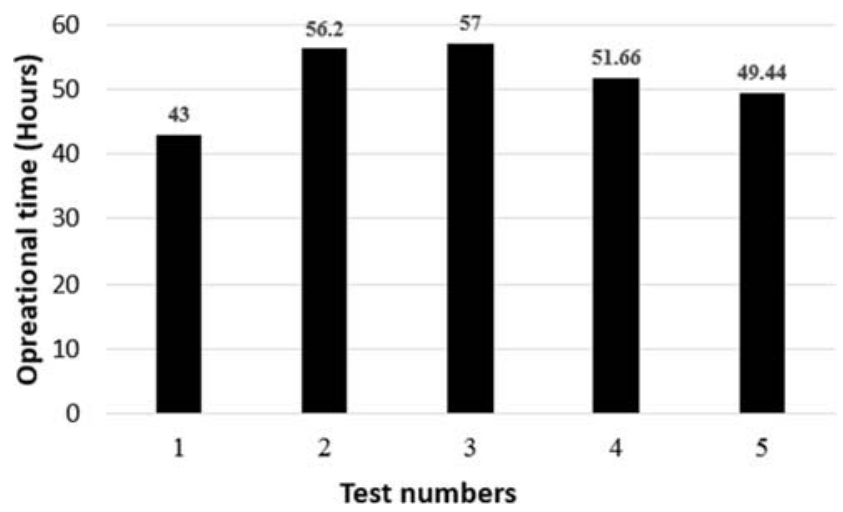

Fig. 125 tests using optimisation setting for 3D printed gears
In the GA optimisation process, 200 iterations were selected due to the decrease in computational time and convergence towards an optimised solution. Each iteration has a population of 50. The plot on the solid line represents the average error corresponding to the test data, and the dotted line represents the best fit corresponding to the test data from the wear test rig. It is shown that average error was decreased from around 23 to $10 \%$; moreover, best fit was improved from 10 to less than $5 \%$, respectively. Hence, it can be shown that applying GA can increase the efficiency and accuracy of the ANN regression model (Fig. 8).

Figure 9 shows the comparison of the prediction of each model and test performed by wear test rig. Compared with evaluation methods such as Pearson's $r$ and $R$-squared, it is shown that leave-one cross validation applied to the GA-based ANN model provides better accuracy compared with a conventional ANN model and the GA-based ANN model. Hence, as a result, leave-one cross validation applied to the GA-based ANN model can provide an efficiently and relatively accurate model to carry out the prediction of performance of $3 \mathrm{D}$ printed nylon spur gears with different manufacturing parameters.

The model reveals (Fig. 10) that printing temperature contributes to the performance of a printed gear by around $22 \%$ in terms of weighting. Printing speed has around a $23 \%$ influence on the performance. Bed temperature contributes an $8.6 \%$ influence on the final result, showing a reduced importance compared with the rest of the parameters. Hence, by using Garson's algorithm, it is possible to identify the most influential parameter regarding gear performance is infill percentage. Conceptually, this result makes sense as it is possible that by increasing infill percentage, the rigidity of gear under loads is increased.

In order to explore the power of the model in predicting optimal gear performance and outputting the $3 \mathrm{D}$ printer parameters required, a simulation was carried out. Figure 8 shows the simulation of 14,256 combinations of different 
parameters. In this simulation, printing temperature is increased from 230 to $275{ }^{\circ} \mathrm{C}$ by $5{ }^{\circ} \mathrm{C}$ each step. Hence, there are 10 data points created for printing temperature instead of 50. Printing speed was increased from 20 to $75 \mathrm{~mm} / \mathrm{s}$ by $5 \mathrm{~mm} / \mathrm{s}$ each step. Hence, there are 12 data points generated. Bed temperature is increased from 30 to $70{ }^{\circ} \mathrm{C}$ with $5^{\circ} \mathrm{C}$ each time, with 9 data points required for the analysis. Infill percentage was increased from 20 to $80 \%$, with 12 data points. As mentioned earlier, there are more than 5 million combinations that could be used in generating test input data; however, errors in the $3 \mathrm{D}$ printing process and errors in the test rig could counter the tolerance of the setting, hence a gap between parameters by factor of 5 could provide relatively accurate results. Simulation was carried out by leave-one cross validation-applied GA-based ANN model. Simulation number 7532 showed $52.07 \mathrm{~h}$ of potential gear performance with $3 \mathrm{D}$ printer settings of a printing temperature of $250{ }^{\circ} \mathrm{C}$, printing speed of $70 \mathrm{~mm} / \mathrm{s}$, bed temperature of $25{ }^{\circ} \mathrm{C}$ and infill percentage of $80 \%$ as shown in Fig. 11.

Validation of this model result was performed by producing a $3 \mathrm{D}$ printed gear using the same settings suggested by the ANN optimisation. A total of 5 pairs of gears were printed and tested on the wear test rig, with the results shown in Fig. 12. The results showed that the 5 tests yielded an average performance of $51.46 \mathrm{~h}$, which was very close to the ANN simulation value of $52.07 \mathrm{~h}$. Hence, optimisation simulation could be considered as a valid simulation.

Previous paper has carried out the analysis of 5 different nylon 3D printing materials. There are 5 different materials that have been printed and tested including Nylon 618, Nylon 645, alloy 910 onyx and Markforged nylon filaments. Nylon 618 3D printed gear provided best wear performance among 5 different 3D printing filament materials. Research shows that the different mechanical performance between nylon filaments was caused by differences in crystallinity and uniqueness of the FDM process. Another outstanding behaviour of Nylon 618 was shown in SEM (scanning electron microscopy); result shows dramatically different wear behaviour for the $3 \mathrm{D}$ printed gears when compared with the literature reports of injection-moulded gears. Furthermore, Nylon 618 material has outstanding thermal performance of gears during wear tests and together with SEM, which was used to analyse gear failure mechanisms. The performance results showed that gears 3D printed using Nylon 618 actually performed better than injection-moulded Nylon 66 gears when low to medium torque was applied. Associating with the result of optimal 3D printer setting, it is believed that by improving the manufacturing procedure, the performance of Nylon 618 was further enhanced. By applying machine learning method to manufacturing $3 \mathrm{D}$ printed gears could dramatically increase the mechanical behaviour of 3D printed part in a highly dynamic criterion.

\section{Conclusion}

In this paper, a set of experimental data has been designed by the Sobol sequence, providing relatively higher tolerance and covering a much larger range of input data with minimal test data being required. Four 3D printing parameters were selected via specific requirements of polymer gears which require rigidity and light weight. A prediction model of 3D printed gears has been carried out with three models including an ANN model, a GA-based ANN model and a leave-one cross validation-applied GAbased ANN model. The results show that all models provide relatively accurate predictions and provide satisfactory fitting to the test data. A leave-one cross validation-applied model provides the strongest correlation with test results, with Pearson's $r$ equal to 0.97 and $R^{2}$ equal to 0.956 , respectively. Moreover, by simulating an experiment, the printing parameters have been optimised to increase the performance of the $3 \mathrm{D}$ printed polymer gears. The results suggest an optimised setting of the $3 \mathrm{D}$ printer as follows: printing temperature is equal to $250{ }^{\circ} \mathrm{C}$, a printing speed of $70 \mathrm{~mm} / \mathrm{s}$, a bed temperature of $25^{\circ} \mathrm{C}$ and the infill percentage is $80 \%$. The operational time of the resultant $3 \mathrm{D}$ printed polymer gear was increased more than 3 times compared with the gears produced using the default print settings. Sensitivity analysis performed by Garson's algorithm indicated that infill percentage has the most influence on the performance of a $3 \mathrm{D}$ printed gear, and bed temperature has the least influence on the test result.

\section{Limitations and future scope}

Due to the unique characteristic of the ANN process, true correlation between each parameter was not fully studied. Moreover, more data points added to the model could increase the accuracy of the simulation. There are several possible directions based on this research. Firstly, to carry out the study of the polymer molecular structure to explain the influence of different parameter settings. Secondly, to investigate several other 3D printed materials in order to understand the correlation between different materials and creating model to predict the performance of gears produced by using different materials and elicit the required print parameters.

Open Access This article is licensed under a Creative Commons Attribution 4.0 International License, which permits use, sharing, adaptation, distribution and reproduction in any medium or format, as long as you give appropriate credit to the original author(s) and the source, provide a link to the Creative Commons licence, and indicate if changes were made. The images or other third party material in this article are included in the article's Creative Commons licence, unless indicated otherwise in a credit line to the material. If material is not included in the article's Creative Commons licence and your intended use is not permitted by statutory regulation or exceeds the permitted use, you will need to obtain permission directly from the copyright holder. To view a copy of this licence, visit http://creativecommons.org/licenses/by/4.0/. 


\section{References}

1. Zhang Y, Purssell C, Mao K, Leigh S (2020) A physical investigation of wear and thermal characteristics of 3D printed nylon spur gears. Tribol Int 141:105953

2. Mao K, Li W, Hooke C, Walton D (2009) Friction and wear behaviour of acetal and nylon gears. Wear 267(1-4):639-645

3. Hu Z, Mao K (2017) An investigation of misalignment effects on the performance of acetal gears. Tribol Int 116:394-402

4. Hooke C, Mao K, Walton D, Breeds A, Kukureka S (1993) Measurement and prediction of the surface temperature in polymer gears and its relationship to gear wear. J Tribol 115(1):119-124

5. Gauvin R, Patrick G, Henry Y (1984) Maximum surface temperature of the thermoplastic gear in a non-lubricated plastic/steel gear pair. Ecole Polytechnique de Montreal. J Manuf Process 20-27

6. Mao K (2007) A numerical method for polymer composite gear flash temperature prediction. Wear 262(11-12):1321-1329

7. Berman B (2012) 3-D printing: the new industrial revolution. Bus Horiz 55(2):155-162

8. Wohlers T (2015) Additive manufacturing and 3D printing state of the industry. Wohlers Associates, Fort Collins ISBN 978-0 9913332-1-9; 2013

9. Chacón J, Caminero MA, García-Plaza E, Núñez PJ (2017) Additive manufacturing of PLA structures using fused deposition modelling: effect of process parameters on mechanical properties and their optimal selection. Mater Des 124:143-157

10. Gomez-Gras G, Jerez-Mesa R, Travieso-Rodriguez JA, LlumaFuentes J (2018) Fatigue performance of fused filament fabrication PLA specimens. Mater Des 140:278-285

11. Kuznetsov VE, Solonin AN, Tavitov AG, Urzhumtcev OD, Vakulik AH (2019) Increasing strength of FFF 3D printed parts by influencing on temperature-related parameters of the process. Rapid Prototyp J. https://doi.org/10.1108/RPJ-01-2019-0017

12. Prajapati H, Ravoori D, Woods RL, Jain A (2018) Measurement of anisotropic thermal conductivity and inter-layer thermal contact resistance in polymer fused deposition modeling (FDM). Addit Manuf 21:84-90

13. Bellehumeur C, Li L, Sun Q, Gu P (2004) Modeling of bond formation between polymer filaments in the fused deposition modeling process. J Manuf Process 6(2):170-178

14. Sun Q, Rizvi G, Bellehumeur C, Gu P (2008) Effect of processing conditions on the bonding quality of FDM polymer filaments. Rapid Prototyp J 14(2):72-80

15. Wang M, Zhu Z, Dong Y, Zhou L (2017) Study of mixed-mode I/II fractures using single cleavage semicircle compression specimens under impacting loads. Eng Fract Mech 177:33-44

16. Deng L, Feng B, Zhang Y (2018) An optimization method for multi-objective and multi-factor designing of a ceramic slurry: combining orthogonal experimental design with artificial neural networks. Ceram Int 44(13):15918-15923

17. Koeppe A, Padilla CAH, Voshage M, Schleifenbaum JH, Markert B (2018) Efficient numerical modeling of 3D-printed lattice-cell structures using neural networks. MFGLET 15:147-150

18. Delli U, Chang S (2018) Automated process monitoring in 3D printing using supervised machine learning. Procedia Manuf 26:865-870

19. Li W, Lin W, Yu J (2016) Predicting contact characteristics for helical gear using support vector machine. Neurocomputing 174: 1156-1161

20. Sun S, Wang S, Wang Y, Lim TC, Yang Y (2018) Prediction and optimization of hobbing gear geometric deviations. Mech Mach Theory 120:288-301

21. Samanta B (2004) Gear fault detection using artificial neural networks and support vector machines with genetic algorithms. Mech Syst Signal Process 18(3):625-644
22. Hadavandi E, Shahrabi J, Hayashi Y (2016) SPMoE: a novel subspace-projected mixture of experts model for multi-target regression problems. Soft Comput 20(5):2047-2065

23. Melki G, Cano A, Kecman V, Ventura S (2017) Multi-target support vector regression via correlation regressor chains. Inf Sci 415:53-69

24. Sobol' IM (1967) On the distribution of points in a cube and the approximate evaluation of integrals. Zh Vychisl Mat Mat Fiz 7(4): 784-802

25. Savine A (2018) Modern computational finance: AAD and parallel simulations. John Wiley \& Sons

26. Wang S-C (2003) Artificial neural network. Interdisciplinary computing in java programming. Springer, Boston, MA, p 81-100

27. Ye Z, Kim MK (2018) Predicting electricity consumption in a building using an optimized back-propagation and LevenbergMarquardt back-propagation neural network: case study of a shopping mall in China. Sustain Cities Soc 42:176-183

28. Vogl TP, Mangis J, Rigler A, Zink W, Alkon D (1988) Accelerating the convergence of the back-propagation method. Biol Cybern 59(4-5):257-263

29. Kshirsagar AP, Rathod MN (2012) Article: artificial neural network. IJCA Proceedings on National Conference on Recent Trends in Computing 2:12-16

30. Quinlan JR (2014) C4. 5: programs for machine learning. Elsevier

31. Mohamad ET, Armaghani DJ, Momeni E, Yazdavar A, Ebrahimi M (2018) Rock strength estimation: a PSO-based BP approach. Neural Comput \& Applic 30(5):1635-1646

32. Dormishi A, Ataei M, Khaloo Kakaie R, Mikaeil R, Shaffiee HS (2019) Performance evaluation of gang saw using hybrid ANFISDE and hybrid ANFIS-PSO algorithms. J Min Reclam Env 10(2): 543-557

33. Babanouri N, Nasab SK, Sarafrazi S (2013) A hybrid particle swarm optimization and multi-layer perceptron algorithm for bivariate fractal analysis of rock fractures roughness. Int J Rock Mech Min Sci 60:66-74

34. Holland JH (1992) Adaptation in natural and artificial systems: an introductory analysis with applications to biology, control, and artificial intelligence. MIT Press

35. Chipperfield AJ, Fleming P, Pohlheim H (1994) Genetic algorithm toolbox: for use with MATLAB; user's guide (version 1.2): University of Sheffield, Department of Automatic Control and Systems Engineering

36. Simpson AR, Dandy GC, Murphy LJ (1994) Genetic algorithms compared to other techniques for pipe optimization. J Water Resour Plan Manag 120(4):423-443

37. Jadav K, Panchal MP (2012) Optimizing weights of artificial neural networks using genetic algorithms. Int J Adv Res Comput Sci Electron Eng 1(10):47-51

38. Vose MD (1999) The simple genetic algorithm: foundations and theory. MIT Press

39. Houck CR, Joines J, Kay MG (1995) A genetic algorithm for function optimization: a Matlab implementation. Ncsu-ie tr 95(09):1-10

40. Wong T-T (2015) Performance evaluation of classification algorithms by k-fold and leave-one-out cross validation. Pattern Recogn 48(9):2839-2846

41. Garson GD (1991) Interpreting neural-network connection weights. AI Expert 6(4):46-51

42. Momeni E, Nazir R, Armaghani DJ, Maizir H (2014) Prediction of pile bearing capacity using a hybrid genetic algorithm-based ANN. Measurement 57:122-131

43. Goh AT (1995) Back-propagation neural networks for modeling complex systems. Artif Intell Eng 9(3):143-151

44. Lee Rodgers J, Nicewander WA (1988) Thirteen ways to look at the correlation coefficient. Am Stat 42(1):59-66

45. Stigler SM (1989) Francis Galton's account of the invention of correlation. Stat Sci 73-79 